\title{
Escarabeídeos (Coleoptera: Scarabaeidae) de campo e floresta da Reserva Biológica de São Donato, Rio Grande do Sul, Brasil
}

\author{
Vinícius da Costa Silva ${ }^{1 *}$ \\ Rafael Parcianello Cipolatto ${ }^{1}$ \\ Arthur Diesel Abegg 1 \\ Conrado Mario da Rosa ${ }^{1}$ \\ Pedro Giovâni da Silva ${ }^{2}$ \\ Rocco Alfredo Di Mare ${ }^{1}$ \\ ${ }^{1}$ Universidade Federal de Santa Maria \\ Departamento de Biologia, Laboratório de Biologia Evolutiva \\ Avenida Roraima, 1000, Camobi, CEP 97105-900, Santa Maria - RS, Brasil \\ ${ }^{2}$ Programa de Pós-Graduação em Ecologia \\ Universidade Federal de Santa Catarina, Florianópolis - SC, Brasil \\ * Autor para correspondência \\ silvavinicius92@gmail.com
}

Submetido em 17/03/2014

Aceito para publicação em 29/09/2014

\section{Resumo}

O objetivo deste trabalho foi comparar a riqueza, abundância e diversidade das espécies de Scarabaeidae em duas fitofisionomias (campo e floresta) e verificar se a variação sazonal ao longo do ano está relacionada com variáveis climáticas. O estudo foi desenvolvido na Reserva Biológica de São Donato, Rio Grande do Sul, Brasil (bioma Pampa). As coletas foram realizadas através de armadilhas de queda, entre janeiro de 2012 e janeiro de 2013. Foram coletados 125 indivíduos e identificados seis gêneros e 11 espécies de quatro subfamílias, sendo 54 indivíduos de cinco espécies encontradas no campo e 71 indivíduos de oito espécies na floresta. As espécies mais abundantes foram Ataenius picinus Harold, 1868, Canthon lividus Blanchard, 1845 e Leucothyreus flavipes Eschscholtz, 1822, que representaram juntas $86,4 \%$ do total de indivíduos capturados. O verão apresentou o maior número de indivíduos (78) e a primavera o maior número de espécies (nove). A diferença na estrutura do ambiente (e clima associado) e provavelmente a disponibilidade diferenciada de recurso alimentar entre as duas fitofisionomias devem ser fatores limitantes e decisivos para a ocorrência de cada subfamília em determinado habitat, uma vez que várias espécies foram restritas a determinada fitofisionomia ou época do ano.

Palavras-chave: Besouros; Campo; Distribuição; Floresta; Sazonalidade

\section{Abstract}

Forest and field abundance of Scarab beetles (Coleoptera: Scarabaeidae) in the São Donato Biological Reserve, Rio Grande do Sul, Brazil. The objective of this work was to compare the species richness, abundance and diversity of Scarabaeidae beetles in two types of habitats (field and forest), and to 
assess whether their seasonal variation is related to climatic variables. This study was conducted in the São Donato Biological Reserve, Rio Grande do Sul state, Brazil (Pampa biome). Beetles were collected using pitfall traps between January 2012 and January 2013. A total of 125 individuals were collected, of which six genera and 11 species from four subfamilies of Scarabaeidae were identified. 54 individuals of five species were collected from the field, and 71 individuals of eight species were collected from the forest. The most abundant species were Ataenius picinus Harold, 1868, Canthon lividus Blanchard, 1845 and Leucothyreus flavipes Eschscholtz, 1822, which together accounted for $86.4 \%$ of all individuals captured. The highest total number of individuals was collected in summer (78), and the highest number of species was collected in spring (9). Differences in environmental structure (and associated climate) and food resource availability may be decisive and limiting factors for beetle occurrence in forest versus field areas, as various species were restricted to a specific habitat type or season.

Key words: Distribution; Field; Forest; Scarab beetles; Seasonality

\section{Introdução}

Atualmente existem cerca de 30.000 espécies de escarabeídeos catalogadas em todo o mundo, distribuídas em 19 subfamílias (BOUCHARD et al., 2011). Estes insetos desempenham diversas funções ecológicas importantes para a manutenção e regulação dos ecossistemas onde estão inseridos, pois possuem espécies que auxiliam na decomposição de material orgânico, dispersão de sementes, controle biológico e polinização, enquanto outras espécies são citadas como pragas de culturas agrícolas (HALFFTER; MATTHEWS, 1966; BOOTH et al., 1990; HANSKI; CAMBEFORT, 1991), tendo grande importância econômica no contexto de agroecossistemas.

Devido às suas adaptações alimentares, Scarabaeidae faz parte de um grupo importante de detritívoros nos ecossistemas, uma vez que utilizam o solo para alocação de recurso, abrigo e nidificação (HALFFTER; MATTHEWS, 1966; HALFFTER; EDMONDS, 1982). Dessa forma, atuam na ciclagem de nutrientes, na aeração do solo, como dispersores secundários de sementes e redutores de parasitas presentes nas fezes dos mamíferos (ANDRESEN, 2002). Segundo Moura (2003), estudos realizados com a família Scarabaeidae no estado do Rio Grande do Sul são poucos e muitos aspectos como comportamento, distribuição, taxonomia e sistemática são desconhecidos para a maioria das espécies, quando comparados a outras famílias de Coleoptera.

Em grupos de organismos onde a competição interespecífica é forte, como em várias subfamílias de
Scarabaeidae, pode-se esperar a presença de associações de espécies com grau alto de fidelidade por um biótopo ou fitofisionomia em particular (DRISCOLL; WEIR, 2005). Deste modo, mudanças espaciais na composição de espécies de Scarabaeidae podem estar relacionadas a aspectos ambientais da variação estrutural dos habitats. Assim, estudos sobre essa fauna em tipos diferentes de ambientes podem auxiliam na melhor compreensão da dinâmica das comunidades e do funcionamento dos ecossistemas.

Diante da importância dos escarabeídeos para os ecossistemas, juntamente com a escassez de estudos sobre o grupo no estado do Rio Grande do Sul, desenvolveu-se o presente trabalho com o objetivo de conhecer as espécies da família Scarabaeidae da Reserva Biológica de São Donato, oeste do estado, em duas fitofisionomias diferentes (campo e floresta), levando em consideração a sazonalidade dos insetos e a heterogeneidade ambiental local. A riqueza de espécies, a abundância e a diversidade foram comparadas entre as fitofisionomias a fim de investigar a influência do tipo de ambiente na distribuição das espécies de Scarabaeidae. A distribuição das espécies foi correlacionada com variáveis climáticas (temperatura média, horas de insolação e umidade relativa do ar) a fim de investigar a sazonalidade do grupo. Com base nas características do grupo estudado, espera-se que campo e floresta apresentem faunas distintas e que a distribuição temporal das espécies de Scarabaeinae seja relacionada com as variáveis climáticas ao longo do período de estudo. 


\section{Material e Métodos}

O estudo foi realizado na Reserva Biológica de São Donato (REBio) localizada entre os municípios de Itaqui e Maçambará (2911'15'S; 56 03'45”O), na região fisiográfica da Campanha dentro do bioma Pampa no estado do Rio Grande do Sul, Brasil (Figura 1). O objetivo principal da reserva é o de proteger o banhado São Donato. A REBio possui área de 4.392 ha e está inserida nos limites da Bacia Hidrográfica do baixo curso do Rio Butuí, afluente do Rio Uruguai (FUNDAÇÃO ZOOBOTÂNICA DO RIO GRANDE DO SUL, 2002), sendo a última formação de banhado restante no estado. Banhado no Rio Grande do Sul designa áreas alagadas temporariamente ou permanentemente, enquanto no restante do país estas áreas são referidas como brejo, pântano, pantanal, charco, varjão e alagado, entre outros (BURGER, 2000).
A temperatura média anual na região é de aproximadamente $20^{\circ} \mathrm{C}$ e a precipitação pluviométrica varia entre 1.500 e $1.650 \mathrm{~mm}$ anuais. A geomorfologia predominantemente é formada por colinas (coxilhas) extensas e com baixa declividade (planas) caracterizando um relevo de planícies (UNIVERSIDADE FEDERAL DE SANTA MARIA, 2007). A REBio está inserida na região da savana/estépica, incluindo grandes áreas úmidas, tabuleiros, florestas-de-galeria e capões. Entre as espécies arbóreas é comum a presença de Parapiptadenia rigida (Benth.) Brenan (angico-vermelho), Ficus sp. (figueira), Salix humboldtiana Willd. (salgueiro) e Syagrus romanzoffiana (Cham.) Glassman (gerivá) (BACKES, 2012). A vegetação do banhado é composta predominantemente por densas formações da ciperácea Scirpus giganteus (Kunth) H.Pfeiff, que ocorre associada ou intercalada a várias outras plantas palustres, sobretudo

FIGURA 1: A) Localização da Reserva Biológica São Donato no Rio Grande do Sul; B) Limites da Reserva e localização das áreas amostradas; C) área de campo; D) área de floresta.

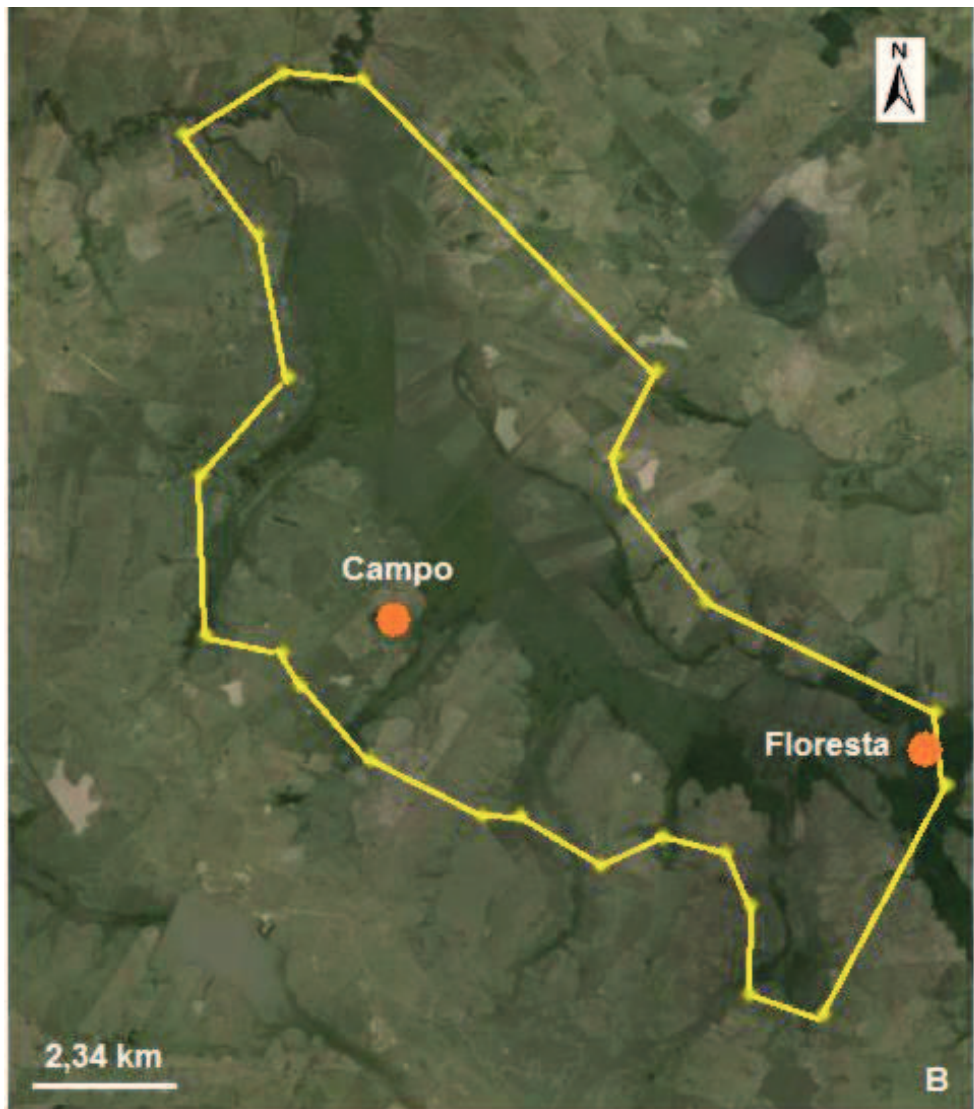

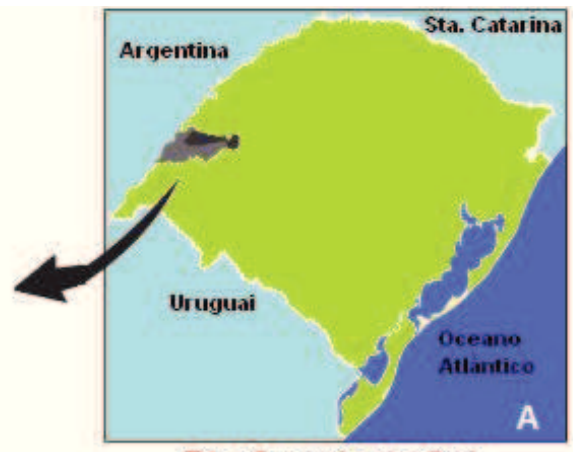

Rio Grande do Sul
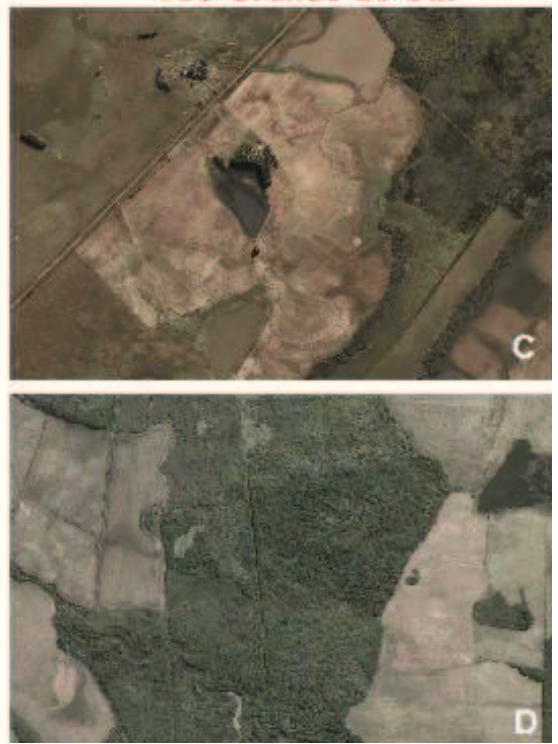
Cyperus giganteus Vahl, (Cyperaceae), Thalia geniculata Linnaeus, (Marantaceae), Panicum grumosum Nees von Esenbeck e $P$. prionitis Nees von Esenbeck (Poaceae) (BENCKE, 2004). A REBio, embora seja uma unidade de conservação, apresenta alterações ambientais notáveis, especialmente em relação à transformação de suas paisagens naturais para o cultivo de lavouras. Pesquisa realizada entre 1990 e 2010 revela uma perda de $32 \%$ de suas paisagens naturais, principalmente para as áreas de lavoura que no mesmo período cresceram $31,25 \%$ (RIGHI, 2012).

O estudo foi realizado em dois tipos de fitofisionomias (Figura 1): campo e floresta nativa. A fitofisionomia de campo (2901'43.2”S; 56 $6^{\circ} 11^{\prime} 40.6$ "O) é composta por vegetação rasteira, desprovida de plantas arbóreas de grande porte, sendo, neste caso, utilizada como área de pastoreio eventual para bovinos. A fitofisionomia de floresta ( $29^{\circ} 02^{\prime} 46.7^{\prime}$ 'S; 56 04 '48.7'O) possui área com alta densidade de árvores de médio e grande porte, o que dificulta o acesso dos bovinos ao interior da mesma. As duas fitofisionomias estavam distantes cerca de $11 \mathrm{~km}$ entre si e ambas apresentavam altitude aproximada de $70 \mathrm{~m}$.

As coletas foram realizadas com armadilhas de queda sem atrativos (AQUINO, 2001), confeccionadas com garrafas plásticas de dois litros, recortadas na parte superior e enterradas com a borda ao nível do solo. Sobre cada armadilha foi colocado um prato descartável sustentado por arames para proteção contra a chuva. No interior de cada recipiente coletor foi adicionado 300 $\mathrm{mL}$ de solução conservante (água, formol a $3 \%$ e sabão líquido). Em cada fitofisionomia as armadilhas foram dispostas em oito transectos distanciados $20 \mathrm{~m}$ entre si. Cada transecto continha cinco armadilhas, distantes $10 \mathrm{~m}$ entre si, totalizando 40 armadilhas em cada tipo de habitat.

As amostragens foram realizadas entre de janeiro de 2012 e janeiro de 2013. As armadilhas permaneceram abertas por 15 dias durante cada período de amostragem, sendo as coletas dos exemplares realizadas uma vez em cada estação do ano, totalizando quatro coletas ao longo do ano. As amostras recolhidas foram levadas para o Laboratório de Biologia Evolutiva da Universidade
Federal de Santa Maria (UFSM), onde foram triadas e os escarabeídeos identificados ao menor nível taxonômico possível. As espécies foram identificadas através da comparação com material depositado na coleção entomológica do Laboratório. O material coletado encontra-se tombado no mesmo local.

Curvas de acumulação de espécies foram utilizadas para avaliar suficiência amostral e comparar o acréscimo de espécies ao longo do período de estudo entre os ecossistemas avaliados (MORENO, 2001) usando o programa EstimateS 9. A riqueza de espécies, abundância e diversidade foram comparadas através de teste $t$ de Student. O índice de diversidade de ShannonWiener $\left(\mathrm{H}^{\prime}\right)$, que considera igual peso às espécies raras e abundantes, foi utilizado para investigar a diversidade de escarabeídeos entre as fitofisionomias. O número de espécies e de indivíduos coletados no campo e na floresta foram submetidos ao teste Shapiro-Wilk para verificar a normalidade antes da realização do teste $t$ (SHAPIRO; WILK, 1965). Este teste não-paramétrico é robusto e recomendado para avaliação da normalidade quando o número de repetições é menor do que 30.

Os índices de dominância de Simpson (D), que é caracterizado por ser sensível a mudanças na composição das espécies mais abundantes (PEET, 1974), assim como o de Berger-Parker foram utilizados para verificar a dominância de espécies em cada fitofisionomia (MORENO, 2001). Como forma de aferir estimativas do número de espécies esperado para a área de estudo, foram calculados os valores de quatro estimadores de riqueza não paramétricos (Jackknife 1, Jackknife 2, Chao 2 e Bootstrap). O teste de Qui-quadrado foi utilizado para testar a existência de diferenças significativas entre os valores estimados e observados.

As variáveis climáticas de temperatura média, tempo médio de insolação diário e umidade relativa média do ar foram coletadas durante os períodos de amostragem e relacionadas com o número de indivíduos capturados através da correlação de Pearson e análise de regressão linear. 


\section{Resultados}

Foram coletados 125 indivíduos distribuídos em seis gêneros e 11 espécies de quatro subfamílias de Scarabaeidae: Aphodiinae - 83 indivíduos (66,4\% do total), Scarabaeinae - 27 indivíduos $(21,6 \%)$, Rutelinae - 10 indivíduos $(8,0 \%)$ e Dynastinae - cinco indivíduos $(5,0 \%)$ (Tabela 1). Somente Dynastinae não foi encontrada nas duas fitofisionomias estudadas. As curvas de acumulação de espécies mostraram-se em forma ascendente e o número de espécies observadas representou $52 \%$ da riqueza estimada de acordo com os estimadores para o campo e $59 \%$ da riqueza na floresta (Figura 2). Os valores estimados para a riqueza de espécies, tanto para o campo (Chao $2=7,25$; Jackknife $1=8$; Jackknife 2 =9,67; Bootstrap $=6,33$ ), quanto para a floresta (Chao $2=10,5$; Jackknife $1=11,75$; Jackknife $2=13,58$; Bootstrap $=9,71$ ), não foram estatisticamente diferentes, quando comparados com os valores obtidos, através do teste de Qui-quadrado.
As espécies mais abundantes foram Ataenius picinus Harold, 1868 (66,4\%) (Aphodiinae), Canthon lividus Blanchard, 1845 (12,8\%) (Scarabaeinae) e Leucothyreus flavipes Eschscholtz, 1822 (7,2\%) (Rutelinae), que representaram juntas $86,4 \%$ do total de indivíduos capturados. Canthon chalybaeus Blanchard, 1845, Canthon rutilans cyanescens Harold, 1868, Canthon lividus, Canthon rutilans rutilans Castelnau, 1840, Deltochilum brasiliense (Castelnau, 1840) e Geniates barbatus Kirby, 1808 foram registradas apenas na fitofisionomia de floresta. Canthon luctuosus Harold, 1868, Canthon mutabilis Lucas, 1857 e Euetheola humilis Burmeister 1847 foram encontradas exclusivamente na fitofisionomia campestre. Somente A. picinus e $L$. flavipes foram amostradas nos dois tipos de fitofisionomias.

Ao longo de todo o estudo, 54 (43,2\%) indivíduos de cinco espécies foram capturados no campo e $71(56,8 \%)$ indivíduos de oito espécies na floresta. O número médio de espécies por transecto diferiu entre as fitofisionomias

TABELA 1: Espécies de Scarabaeidae amostradas com armadilhas de queda em fitofisionomias de campo e de floresta na Reserva Biológica de São Donato, Rio Grande do Sul, Brasil, entre janeiro de 2012 e janeiro de 2013.

\begin{tabular}{|c|c|c|c|c|c|c|c|}
\hline \multirow{2}{*}{ Subfamília/Espécie } & \multicolumn{2}{|c|}{ Fitofisionomia } & \multirow{2}{*}{ Total } & \multicolumn{4}{|c|}{ Estação } \\
\hline & Campo & Floresta & & Inverno & Outono & Primavera & Verão \\
\hline \multicolumn{8}{|l|}{ Aphodiinae } \\
\hline Ataenius picinus Harold, 1868 & 46 & 37 & 83 & - & 2 & 16 & 65 \\
\hline \multicolumn{8}{|l|}{ Dynastinae } \\
\hline Euetheola humilis Burmeister 1847 & 5 & - & 5 & - & - & 5 & - \\
\hline \multicolumn{8}{|l|}{ Rutelinae } \\
\hline Geniates barbatus Kirby, 1808 & - & 1 & 1 & - & - & - & 1 \\
\hline Leucothyreus flavipes Eschscholtz, 1822 & 1 & 8 & 9 & - & - & 1 & 8 \\
\hline \multicolumn{8}{|l|}{ Scarabaeinae } \\
\hline Canthon chalybaeus Blanchard, 1845 & - & 1 & 1 & - & - & 1 & - \\
\hline Canthon lividus Blanchard, 1845 & - & 16 & 16 & - & - & 16 & - \\
\hline Canthon mutabilis Lucas, 1857 & 1 & - & 1 & - & - & 1 & - \\
\hline Canthon luctuosus Harold, 1868 & 1 & - & 1 & - & - & 1 & - \\
\hline Canthon rutilans cyanescens Harold, 1868 & - & 1 & 1 & - & - & 1 & - \\
\hline Canthon rutilans rutilans Castelnau, 1840 & - & 6 & 6 & - & - & 3 & 3 \\
\hline Deltochilum brasiliense (Castelnau, 1840) & - & 1 & 1 & - & - & - & 1 \\
\hline Número de indivíduos & 54 & 71 & 125 & 0 & 2 & 45 & 78 \\
\hline Número de espécies & 5 & 8 & 11 & 0 & 1 & 9 & 5 \\
\hline
\end{tabular}


FIGURA 2: Curvas de acumulação de espécies de Scarabaeidae registradas em campo e floresta na Reserva Biológica São Donato entre janeiro de 2012 e janeiro de 2013.

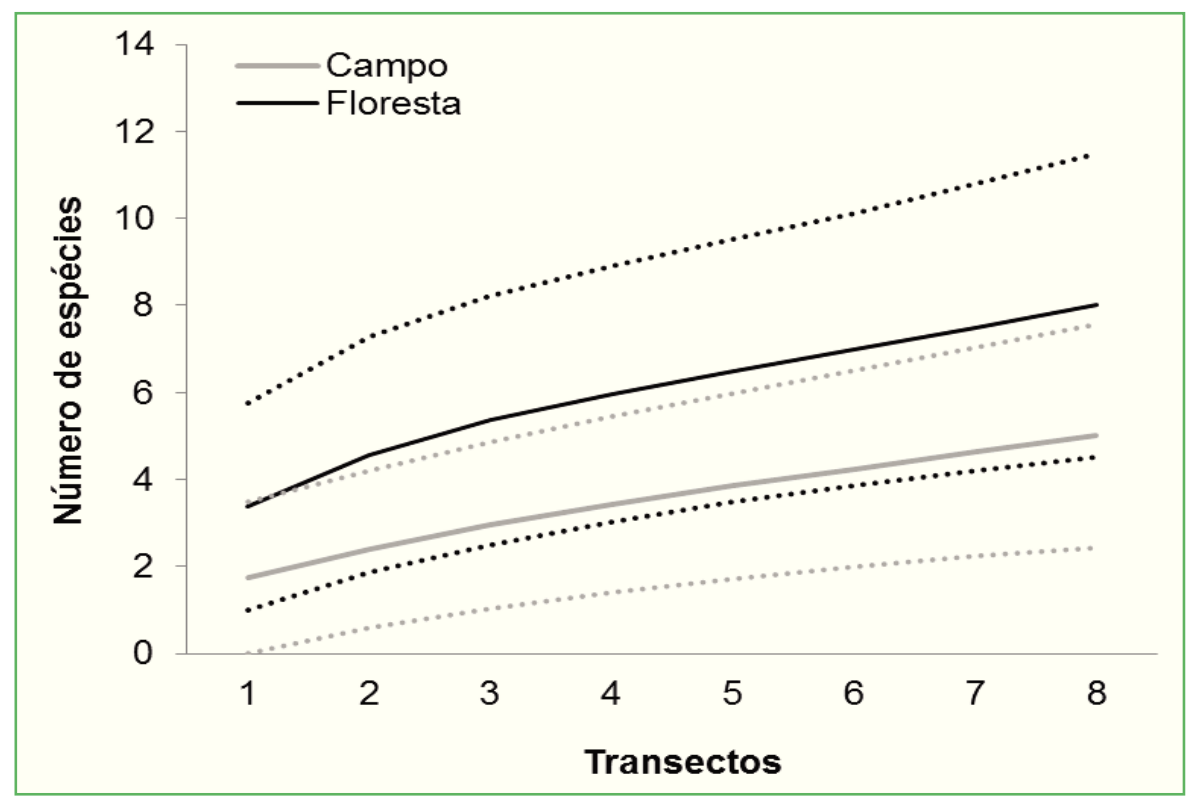

(teste $t=-3,326, \mathrm{p}=0,009$ ), e a floresta apresentou os maiores valores. O número médio de indivíduos por transecto não diferiu entre as fitofisionomias (teste $t=-1,049, \mathrm{p}=0,319)$.

O índice de diversidade de Shannon-Wiener apontou a floresta como a área de maior diversidade (campo: H' $=0,542$; floresta: $\mathrm{H}^{\prime}=1,32$ ), sendo estes valores estatisticamente diferentes (teste $t=-4,189$; $\mathrm{p}<0,05)$. Os índices de dominância do campo apresentaram maiores valores tanto para o índice de Simpson (campo: $\mathrm{D}=0,735$; floresta: $\mathrm{D}=0,343$ ) quanto para o índice de Berger-Parker (campo $=0,852$; floresta $=0,521)$.

Ataenius picinus foi mais abundante no verão (78,3\%) e na primavera (19,3\%) e também a espécie com maior número de capturas no campo $(55,4 \%)$ e na floresta (44,6\%). Canthon lividus (12,8\%), a segunda espécie mais abundante, foi capturada apenas na primavera e na floresta. A terceira espécie mais abundante, L. flavipes $(7,2 \%)$, ocorreu no verão e na primavera em ambas as fitofisionomias estudadas. Deltochilum brasiliense, $G$. barbatus e C. chalybaeus tiveram apenas um indivíduo coletado ao longo do ano, sendo as duas primeiras no verão e a terceira na primavera, respectivamente.

Os dados sazonais de número de indivíduos das espécies identificadas, ao serem correlacionados com os dados de temperatura média, tempo médio de insolação e umidade relativa média do ar, nos dias de captura em cada estação (Tabela 2), apresentaram valores de correlação de 0,91, 0,64 e 0,17 , respectivamente. A análise de regressão linear, utilizada para verificar a influência dos fatores climáticos sobre o número de indivíduos capturados, apresentou os coeficientes de determinação de $0,84,0,42$ e 0,03 , para temperatura média, tempo médio de insolação e umidade relativa média do ar, respectivamente. Estas variáveis quando submetidas ao teste $\mathrm{F}$ (Tabela 2) não apresentaram resultados significativos, embora o coeficiente de determinação para a temperatura média tenha sido alto. 
TABELA 2: Adultos de Scarabaeidae capturados em fitofisionomias de campo (Ca) e de floresta (Fl) na Reserva Biológica de São Donato, e dados de temperaturas médias (Tm), em ${ }^{\circ} \mathrm{C}$, tempo médio de insolação (I), em horas, umidade relativa média do ar (UR), em \%. P: primavera; V: verão; O: outono; I: inverno.

\begin{tabular}{lccccccccccc}
\hline Período & $\mathbf{P}$ & $\mathbf{V}$ & $\mathbf{O}$ & $\mathbf{I}$ & $\mathbf{F}$ & $\mathbf{r}$ & $\mathbf{r}^{\mathbf{2}}$ & $\mathbf{b}$ & $\mathbf{P}_{\mathbf{F}}$ & $\mathbf{t}$ & $\mathbf{P}_{\mathbf{t}}$ \\
\hline Tm & 20,8 & 27,7 & 19,4 & 14,3 & 10,23 & 0,91 & 0,84 & 0,13 & 0,08 & 3,20 & 0,08 \\
$\mathbf{I}$ & 6,0 & 8,0 & 7,1 & 4,5 & 1,42 & 0,64 & 0,42 & 0,03 & 0,36 & 1,19 & 0,35 \\
UR & 47,0 & 78,7 & 62,0 & 72,2 & 0,06 & 0,17 & 0,03 & 0,06 & 0,83 & 0,24 & 0,83 \\
Ca & 13 & 41 & 0 & 0 & - & - & - & - & - & - & - \\
Fl & 32 & 37 & 2 & 0 & - & - & - & - & - & - & - \\
Total & 45 & 78 & 2 & 0 & - & - & - & - & - & - & - \\
\hline
\end{tabular}

Níveis de significância $=0,05 ; \mathrm{b}=$ coeficiente de regressão; $\mathrm{F}=$ Teste $\mathrm{F}_{(1,2)} ; \mathrm{t}_{(\mathrm{n}-2)}=$ teste $t$ de Student; $\mathrm{P}_{\mathrm{F}}$ e $\mathrm{P}_{\mathrm{t}}=\mathrm{p}$-valores associados aos testes $\mathrm{F}$ e $t$.

\section{Discussão}

Os resultados apresentados nas amostragens ao longo de um ano mostraram a distribuição sazonal e espacial dos Scarabaeidae em duas fitofisionomias diferentes, campo e floresta. Estas duas fitofisionomias diferiram apenas no número médio de espécies e algumas delas estiveram restritas à determinada fitofisionomia $\mathrm{e}$ estação do ano. Os escarabeídeos apresentam hábitos e comportamentos variados, onde cada subfamília possui diferentes hábitos alimentares (MARINONI et al., 2001) e estratégias de forrageamento, além de relações com determinados tipos de ambiente. $\mathrm{Na}$ subfamília Aphodiinae, por exemplo, há indivíduos que são detritívoros e coprófagos e muitas espécies estão relacionadas a fezes de bovinos (KOLLER et al., 2007) que são comuns nas áreas campestres do Rio Grande do Sul. Ataenius picinus ocorreu em elevada abundância em ambas as fitofisionomias e possivelmente o rebanho bovino tem ofertado alimento tanto no campo como na floresta. O único dinastíneo coletado, E. humilis, é uma espécie assinalada como praga agrícola tanto na forma larval como na adulta, e ocorreu apenas no campo, onde há plantações de soja e arroz (MARTINS et al., 2000) nas proximidades do local de coleta, o que pode estar relacionado com sua distribuição espacial.

Na subfamília Rutelinae os besouros apresentam dieta herbívora, principalmente de gramíneas e também de matéria vegetal em decomposição (LIMA, 19521956; CARLSON, 1991). Neste sentido, a floresta pode ofertar uma maior quantidade de matéria orgânica em decomposição o que pode ter influenciado a ocorrência de G. barbatus e L. flavipes. A subfamília Scarabaeinae possui besouros detritívoros (coprófagos, necrófagos e saprófagos), e ocorreram tanto no campo como na floresta, podendo estar associados principalmente aos excrementos de mamíferos (SILVA et al., 2012), tanto nativos como de gado. A maioria dos escarabeíneos ocorreu na floresta e muitas dessas espécies preferem ambientes florestados (e.g. C. rutilans cyanescens, C. rutilans rutilans e $D$. brasiliense) enquanto outras ocorrem tanto em florestas como em áreas abertas (SILVA et al., 2011). Além das diferenças estruturais do ambiente, as florestas podem abrigar espécies diferentes de mamíferos em relação às áreas abertas e, consequentemente, ofertar outros tipos de alimento.

Durante o inverno nenhum indivíduo de Scarabaeidae foi capturado. Contudo, para os afodíneos, possivelmente a presença ao longo da maior parte do ano se deve pelo seu ciclo biológico curto. Wegner e Niemczyk (1981) ressaltam que ciclos de curta duração podem favorecer a extensa distribuição temporal dos afodíneos, pois apresentam várias gerações no ano. O menor número de escarabeídeos capturados nos meses que tiveram as menores temperaturas mínimas sugere que temperaturas baixas são limitantes para a atividade dos escarabeídeos no sul do Brasil (SILVA et al., 2013). O clima da região pode afetar diretamente o desenvolvimento e a atividade dos insetos, pois eles não possuem sistema de termorregulação (pecilotérmicos). 
Em contrapartida, as estações com maior número de indivíduos coletados foram verão e primavera, épocas de elevadas temperaturas. Contudo, o número de espécies foi maior na primavera do que no verão, assim como observado por Silva et al. (2013) para a fauna de Scarabaeinae em Santa Maria, na região central do Rio Grande do Sul. A maior riqueza de espécies observada na primavera pode estar relacionada ao período de emergência dos adultos logo após a época de baixas temperaturas (HALFFTER; MATTHEWS, 1966). Freitas et al. (2002) sugerem que coleópteros em geral optam por climas quentes e úmidos para manterem seus ciclos biológicos. No sul do Brasil, o inverno representa um período inapropriado para a reprodução e mesmo para a atividade dos escarabeídeos devido às baixas temperaturas. Muitas das espécies atravessam períodos desfavoráveis na forma de larva, emergindo quando as condições climáticas tornam-se apropriadas (HALFFTER; MATTHEWS, 1966).

A provável diferença microclimática entre as duas fitofisionomias devido à estrutura dos dois ambientes e provavelmente a disponibilidade diferenciada de alimento preferencial das espécies em cada local devem ser os fatores limitantes e decisivos para a ocorrência de cada subfamília (e espécies) em determinado ambiente. Várias espécies estiveram associadas à determinada fitofisionomia ou época do ano. Estes padrões de distribuição espacial e sazonal devem ser mais bem investigados em diferentes escalas espaciais e temporais a fim de elucidar esta importante questão ecológica acerca da estruturação das comunidades biológicas, em especial, dos escarabeídeos, um grupo que desempenha importantes funções ecológicas.

\section{Agradecimentos}

Agradecimento especial ao colega Miguel Machado da Silva pela disponibilização das armadilhas de queda e pela ajuda em campo.

\section{Referências}

ANDRESEN, E. Dung beetles in a Central Amazonian rainforest and their ecological role as secondary seed dispersers. Ecological Entomology, London, v. 27, p. 257-270, 2002.
AQUINO, A. M. Manual para a coleta da macrofauna do solo. Seropédica: EMBRAPA-CNPAB, 2001. 21 p. (Série Documentos, 130).

BACKES, A. Áreas protegidas no estado do Rio Grande do Sul: o esforço para a conservação. São Leopoldo: Instituto Anchietano de Pesquisas, 2012. $131 \mathrm{p}$

BENCKE, G. A. O caboclinho Sporophila zelichi observado no Rio Grande do Sul, Brasil. Ararajuba, Londrina, v. 12, n. 2, p. 88-89, 2004.

BOOTH, R. G.; COX, M. L.; MADGE, R. B. IIE Guides to insects of importance to man: 3. Coleoptera. London: International Institute of Entomology/The Natural History Museum, 1990. 384 p.

BOUCHARD, P.; BOUSQUET, Y.; DAVIES, A. E.; ALONSOZARAZAGA, M. A.; LAWRENCE, J. F.; LYAL, C. H. C.; NEWTON, A. F.; REID, C. A. M.; SCHMITT, M.; SLIPINSKI, A.; SMITH, A. B. T. Family-group names in Coleoptera (Insecta). ZooKeys, Sofia, v. 88, p. 1-972, 2011.

BURGER, M. I. Situação e ações prioritárias para conservação de banhados e áreas úmidas da Zona Costeira. 2000. 60 p. (Relatório de Consultoria).

CARLSON, D. C. Scarabaeidae (Scarabaeoidea). In: STEHR, F. W. (Ed.). Immature insects. Dubuque: Kendall/Hunt Publishing Company, 1991. p. 377-384.

DRISCOLL, D. A.; WEIR, T. Beetle responses to habitat fragmentation depend on ecological traits, habitat condition, and remnant size. Conservation Biology, San Francisco, v. 19, p. 182 194, 2005

FREITAS, F. A.; ZANUNCIO, V. T.; LACERDA, C. M.; ZANUNCIO, C. J. Fauna de Coleoptera coletada com armadilhas luminosas em plantio de Eucalyptus grandis em Santa Bárbara, Minas Gerais. Revista Árvore, Viçosa, v. 26, p. 505-511, 2002.

FUNDAÇÃO ZOOBOTÂNICA DO RIO GRANDE DO SUL. Avaliação da biodiversidade da Reserva Biológica de São Donato. Proposição de limites da Reserva Biológica de São Donato. Porto Alegre: FZB/RS, 2002. 21 p.

HALFFTER, G.; EDMONDS, W. D. The nesting behavior of dung beetles (Scarabaeinae): an ecologic and evolutive approach. México: Man and Biosphere Program UNESCO, 1982. 177 p.

HALFFTER, G.; MATTHEWS, E. G. The natural history of dung beetles of the subfamily Scarabaeinae (Coleoptera: Scarabaeidae). Folia Entomológica Mexicana, Xalapa, v. 12, n. 14, p. 1-312, 1966.

HANSKI, I.; CAMBEFORT, Y. Competition in dung beetles. In: HANSKI, I.; CAMBEFORT, Y. (Ed.). Dung beetle ecology. Princeton: Princeton University Press, 1991. p. 305-329.

KOLLER, W. W.; GOMES, A.; RODRIGUES, S. R.; GOIOZO, P. F. I. Scarabaeidae e Aphodiidae coprófagos em pastagens cultivadas em área do cerrado sul-mato-grossense. Revista Brasileira de Zoociências, Juiz de Fora, v. 9, n. 1, p. 81-93, 2007.

LIMA, A. M. C. Insetos do Brasil, Coleópteros. Vol. 7-10. Rio de Janeiro: Escola Nacional de Agronomia, 1952-1956.

MARINONI, R. C.; GANHO, N. G.; MONNÉ, L. M.; MERMUDES, J. R. M. Hábitos alimentares em Coleoptera (Insecta). Ribeirão Preto: Holos Editora, 2001. 64 p.

MARTINS, J. F. S.; CUNHA, U. S.; OLIVEIRA, J. V.; PRANDO, H. F. Controle de insetos na cultura do arroz irrigado. In: GUEDES, 
J. C.; COSTA, I. F. D.; CASTIGLIONI, E. (Org.). Bases e técnicas do manejo de insetos. Santa Maria: Pallotti, 2000. p. 137-153.

MORENO, C. F. Métodos para medir la biodiversidad. M\&TManuales \& Tesis SEA. Vol. 1. Zaragoza: Gorfi, 2001. 84 p.

MOURA, L. A. Coleópteros. In: FONTANA, C. S.; BENCKE, G. A.; REIS, R. E. (Ed.). Livro vermelho da fauna ameaçada de no Rio Grande do Sul. Porto Alegre: EDIPUCRS, 2003. p. 85-94.

PEET, R. K. The measurement of species diversity. Annual Review of Ecology and Systematics, Palo Alto, v. 5, p. 285-307, 1974.

RIGHI, F. P. A cartografia ambiental como suporte para o estudo das unidades de paisagem: o caso da reserva biológica de São Donato. 2012. 118 f. Dissertação (Mestrado em Geografia Humana) - Universidade de São Paulo, São Paulo. 2012.

SHAPIRO, S. S.; WILK, M. B. An analysis of variance test for normality (complete samples). Biometrika, Oxford, v. 52, n. 3/4, p. 591-611, 1965.

SILVA, P. G.; AUDINO, L. D.; NOGUEIRA, J. M.; MORAES, L. P.; VAZ-DE-MELLO, F. Z. Escarabeíneos (Coleoptera: Scarabaeidae: Scarabaeinae) de uma área de campo nativo no bioma Pampa, Rio Grande do Sul, Brasil. Biota Neotropica, Campinas, v. 12, n. 3, p. 246-253, 2012.
SILVA, P. G.; VAZ-DE-MELLO, F. Z.; DI MARE, R. A. Guia de identificação das espécies de Scarabaeinae (Coleoptera: Scarabaeidae) do município de Santa Maria, Rio Grande do Sul, Brasil. Biota Neotropica, Campinas, v. 11, n. 4, p. 329-345, 2011.

SILVA, P. G.; VAZ-DE-MELLO, F. Z.; DI MARE, R. A. Diversity and seasonality of Scarabaeinae (Coleoptera: Scarabaeidae) in forest fragments in Santa Maria, Rio Grande do Sul, Brazil. Anais da Academia Brasileira de Ciências, Rio de Janeiro, v. 85, n. 2, p. 679-697, 2013.

UNIVERSIDADE FEDERAL DE SANTA MARIA. Atlas geoambiental de São Borja. Santa Maria: UFSM/LAGEOLAM, 2007. 59 p.

WEGNER, G. S.: NIEMCZYK, H. D. Bionomics and phenology of Ataenius spretulus. Annals of the Entomological Society of America, Washington, v. 74, n. 4, p. 374-384, 1981. 